\title{
NUMERICAL MODELING OF TURBULENT SWIRLING TWO-PHASE FLOW IN A CENTRIFUGAL CLASSIFIER
}

\author{
Alexander Shvab ${ }^{1}$, Pavel Zyatikov ${ }^{2}$, a Marina Goyko ${ }^{1}$ \\ ${ }^{1}$ National Research Tomsk State University, 634050 Tomsk, Russia \\ ${ }^{2}$ National Research Tomsk Polytechnic University, 634050 Tomsk, Russia
}

\begin{abstract}
The paper deals with the turbulent swirling flow in the vortex chamber. We study the process of fractional separation of solid particles on the basis of calculating the trajectories. As a result, the probability curves obtained separation Tromp at various regime parameters.
\end{abstract}

In this paper for numerical modeling aerodynamic classifier is selected as a prototype vortex chamber [1], which is a cylindrical region with no rotating wall (Figure 1). In chamber of this type, the swirling flow of gas with the particles of the cross section A-A enters into the swirl chamber and under the influence of differential pressure with the fine fraction of particles go out through the section EE. A large fraction of the particles, due to the effect on it centrifugal force, which dominates over aerodynamic drag force of particles dropped on the peripheral wall of the classifier, and gets into a major product of the fractional division. It is known that the vortex chamber of this type have a fairly large capacity for flow of the carrier medium and solids. However, the efficiency of fractional separation particle size is substantially lower than in air-centrifugal classifier [2]. From our point of view, one reason for the relatively low efficiency of fractional separation of fine powders is significant heterogeneity of the circumferential speed of the field. For its alignment will use the additional rotation of the walls of the vortex chamber with an angular speed $\Omega_{\mathrm{d}}$.

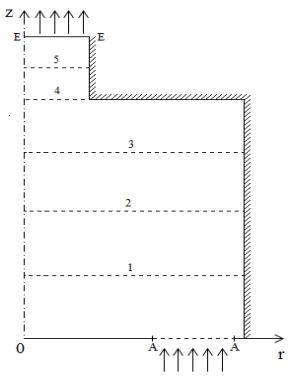

Figure 1. Calculated area.

Thus, aerodynamics of the swirling flow in the zone of separation, shown in Figure 1 will be carried out with considering a rotation of the walls of the vortex chamber. The motion of swirling flow of the carrier medium is described by the Reynolds differential equations in cylindrical coordinates. Since Reynolds equations are not closed, due to the presence of unknown turbulent stress tensor components, you must use the Boussinesq approximation. In view of the above, the system of differential equations Reynolds in a cylindrical coordinate system and in dimensionless form:

\footnotetext{
${ }^{a}$ Corresponding author: impression1994@mail.ru
} 


$$
\begin{aligned}
& \frac{\partial U_{r}}{\partial \tau}+\frac{\partial U_{r}^{2}}{\partial r}+\frac{\partial\left(U_{r} U_{z}\right)}{\partial z}-\frac{1}{\operatorname{Re}} \frac{\partial}{\partial r}\left(1+v_{t}\right) \frac{\partial U_{r}}{\partial r}-\frac{1}{\operatorname{Re}} \frac{\partial}{\partial z}\left(1+v_{t}\right) \frac{\partial U_{z}}{\partial r}=-\frac{\partial P}{\partial r}+\frac{U_{\phi}^{2}}{r}+ \\
& +\frac{1}{\operatorname{Re}}\left(\frac{\partial v_{t}}{\partial r} \frac{\partial U_{r}}{\partial r}+\frac{\partial v_{t}}{\partial z} \frac{\partial U_{z}}{\partial r}\right)-\frac{1}{\operatorname{Re}}\left(1+v_{t}\right) \frac{U_{r}}{r^{2}}-\frac{U_{r}^{2}}{r^{2}}+\frac{1}{\operatorname{Re}}\left(1+v_{t}\right) \frac{\partial U_{r}}{\partial r}, \\
& \frac{\partial U_{z}}{\partial \tau}+\frac{\partial\left(U_{r} U_{z}\right)}{\partial r}+\frac{\partial U_{z}^{2}}{\partial z}-\frac{1}{\operatorname{Re}} \frac{\partial}{\partial r}\left(1+v_{t}\right) \frac{\partial U_{z}}{\partial r}-\frac{1}{\operatorname{Re}} \frac{\partial}{\partial z}\left(1+v_{t}\right) \frac{\partial U_{z}}{\partial z}=-\frac{\partial P}{\partial z}-\frac{U_{r} U_{z}}{r}+ \\
& +\frac{1}{\operatorname{Re}}\left(\frac{\partial v_{t}}{\partial r} \frac{\partial U_{r}}{\partial z}+\frac{\partial v_{t}}{\partial z} \frac{\partial U_{z}}{\partial z}\right)+\frac{1}{\operatorname{Re}}\left(1+v_{t}\right) \frac{\partial U_{z}}{\partial r}, \\
& \frac{\partial U_{\phi}}{\partial \tau}+\frac{\partial\left(U_{r} U_{\phi}\right)}{\partial r}+\frac{\partial\left(U_{z} U_{\phi}\right)}{\partial r}-\frac{1}{\operatorname{Re}} \frac{\partial}{\partial r}\left(1+v_{t}\right) \frac{\partial U_{\phi}}{\partial r}-\frac{1}{\operatorname{Re}} \frac{\partial}{\partial z}\left(1+v_{t}\right) \frac{\partial U_{\phi}}{\partial z}=-2 \frac{U_{r} U_{z}}{r}+ \\
& +\frac{1}{\operatorname{Re}} U_{\phi} \frac{\partial v_{t}}{\partial r}+\frac{1}{\operatorname{Re}}\left(1+v_{t}\right) \frac{\partial U_{\phi}}{\partial r}-\frac{1}{\operatorname{Re}}\left(1+v_{t}\right) \frac{U_{\phi}}{r}, \\
& \frac{\partial U_{r}}{\partial r}+\frac{U_{r}}{r}+\frac{\partial U_{z}}{\partial z}=0
\end{aligned}
$$

dimensionless form of equations is obtained by introducing length scales R0 (the radius of the inlet chamber) and velocity U0 (the average speed at the inlet into the swirl chamber).

To determine the eddy viscosity is used two-parameter ke- $\omega$ turbulence model Wilcox. In a cylindrical coordinate system, taking into account the axial symmetry and in dimensionless form, these equations are as follows:

$$
\begin{aligned}
& \frac{\partial k}{\partial \tau}+\frac{\partial\left(U_{r} k\right)}{\partial r}+\frac{\partial\left(U_{z} k\right)}{\partial z}-\frac{1}{\operatorname{Re}} \frac{\partial}{\partial r}\left(1+v_{t} \sigma^{*}\right) \frac{\partial k}{\partial r}-\frac{1}{\operatorname{Re}} \frac{\partial}{\partial z}\left(1+v_{t} \sigma^{*}\right) \frac{\partial k}{\partial z}=G-\beta^{*} k \omega-\frac{U_{r} k}{r}+\frac{1}{\operatorname{Re} \cdot r} \frac{\partial k}{\partial r} \\
& \frac{\partial \omega}{\partial \tau}+\frac{\partial\left(U_{r} \omega\right)}{\partial r}+\frac{\partial\left(U_{z} \omega\right)}{\partial z}-\frac{1}{\operatorname{Re}} \frac{\partial}{\partial r}\left(1+v_{t} \sigma\right) \frac{\partial \omega}{\partial r}-\frac{1}{\operatorname{Re}} \frac{\partial}{\partial z}\left(1+v_{t} \sigma\right) \frac{\partial \omega}{\partial z}=\gamma G \frac{\omega}{k}-\beta \omega^{2}-\frac{U_{r} \omega}{r}+\frac{1}{\operatorname{Re} \cdot r} \frac{\partial \omega}{\partial r}
\end{aligned}
$$

where

$$
v_{t}=\operatorname{Re} \frac{\omega}{k} ; G=\frac{v_{t}}{\operatorname{Re}}\left\{2\left[\left(\frac{\partial U_{r}}{\partial r}\right)^{2}+\left(\frac{\partial U_{z}}{\partial z}\right)^{2}+\left(\frac{U_{r}}{r}\right)^{2}\right]+\left(\frac{\partial U_{\varphi}}{\partial r}-\frac{U_{\varphi}}{r}\right)^{2}+\left(\frac{\partial U_{\varphi}}{\partial z}\right)^{2}+\left(\frac{\partial U_{z}}{\partial r}+\frac{\partial U_{r}}{\partial z}\right)^{2}\right\}
$$

The constants used in the selected turbulence model are: $\beta^{*}=9 / 100, \beta=3 / 40, \sigma^{*}=1 / 2, \sigma=1 / 2, \gamma=5 / 9$.

From the above it follows that the system of equations (1), (2-3) is closed, and describes a two-phase turbulent swirling fluid in the vortex chamber (Fig. 1).

The solution of the transfer of momentum and continuity equations was conducted in variables "velocity-pressure" by splitting by time pressure and velocity fields. The method is implemented on a staggered grid, which allows for the implementation of the equation of continuity at interior points. For each scalar transport equation in the system of equations velocity-pressure used implicit method of alternating directions in $\Delta$-shape, which has a second-order accuracy in time. To obtain a unique solution you must set the following boundary conditions. On the walls of the chamber for the components of the velocity vector operated Terms of sticking. On the axis of symmetry defined Neumann conditions. At the exit of the vortex chamber for the kinetic energy of pulsation movement and a specific rate of dissipation operated the conditions of Neumann. At the inlet are given constant values for the kinetic energy and eddy viscosity, which are calculated during the experiment. On the solid walls of the kinetic energy is zero, and the specific rate of dissipation of pulsating movement on the walls is determined from the equation of diffusion and dissipation in the transport equation $\omega$.

The reliability of the solutions is verified by comparison with experimental data (Fig. 2B) for the turbulent swirling flow in the channel (Fig. 2A).

A)

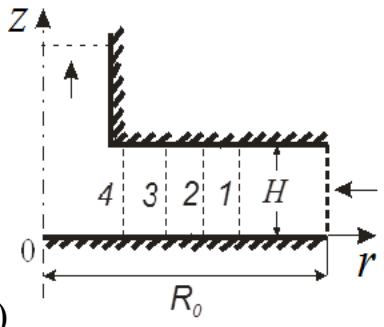

B)

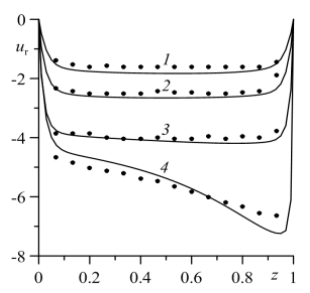

Figure 2. A) current channel and B) comparing the radial velocity component with the experimental data $\mathrm{Re}=2204 ; 1-\mathrm{R} / \mathrm{R} 0=0.6,2-\mathrm{R} / \mathrm{R} 0=0.4,3-$ $\mathrm{R} / \mathrm{R} 0=0.275,4-\mathrm{R} R 0=0.185$. 
Numerical investigations of the peripheral speed of the field have shown that near the walls of the vortex chamber are formed substantial circumferential velocity gradients in the absence of rotation of the walls, which can cause large particles getting into a fine product of the fractional separation. In the case of rotating the walls of chamber the field of circumferential speed substantially aligned not only near the walls of the apparatus, but also throughout the region.

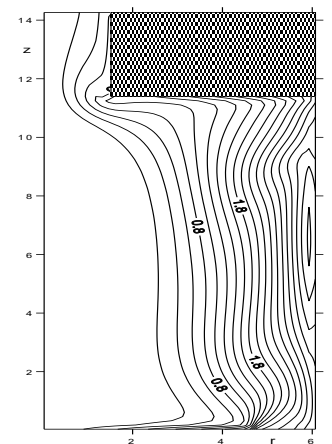

Figure 3. Isolines district component of velocity in the vortex chamber at the parameters $\mathrm{Re}=11000, \mathrm{Rg}=0.5, \mathrm{Rd}=0.5$.

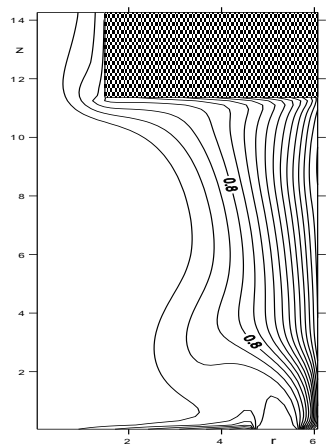

Figure 4. Isolines district component of velocity in the vortex chamber at the parameters $\mathrm{Re}=11000, \mathrm{Rg}=0.5, \mathrm{Rd}=0$.

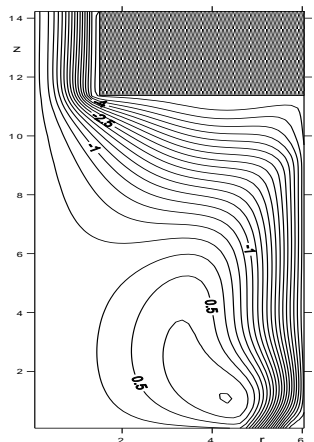

Figure 5. Isolines district component of velocity in the vortex chamber at the parameters $\mathrm{Re}=11000, \mathrm{Rg}=0,5, \mathrm{Rd}=0,5$.

The motion of the dispersed phase is modeled by a set of solutions for the $j$ fraction particles with $k$ starting point. The equations by which may be determined by the trajectory and speed of the particles is given:

$$
\begin{gathered}
\frac{d w_{r}}{d \tau}=\frac{w_{\varphi}^{2}}{r}+\frac{u_{r}-w_{r}}{S t k} \xi, \\
\frac{d w_{\varphi}}{d \tau}=-\frac{w_{r} w_{\varphi}}{r}+\frac{u_{\varphi}-w_{\varphi}}{S t k} \xi, \\
\frac{d w_{z}}{d \tau}=\frac{u_{z}-w_{z}}{S t k} \xi-\frac{1}{F r}, \\
\frac{\Delta r}{w_{r}}=\frac{\Delta z}{w_{z}}=\frac{r \Delta \varphi}{w_{\varphi}}=\Delta \tau,
\end{gathered}
$$

where $\xi$ - coefficient accounting for the deviation of the resistance coefficient of Stokes' law, recorded based on the formula L.S.Klyachko.

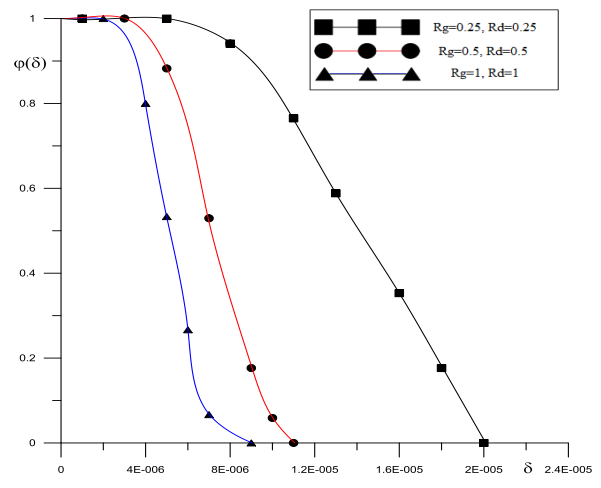

Figure 6. Curve of fractional size separation. 


\section{EPJ Web of Conferences}

\section{References}

1. Ushakov S.G. Inertial separation of dust (M .: Energy. 1974)

2. Zyatikov P.N. Roslyak A.T., Vasenin I.M., Schvab A.V., A.A. Demidenko, Sadretdinov Sh.R, Patent number 2407601, PC V07V7 / 083, A method of air-centrifugal classifying powders and device for its implementation 